\title{
LA-UR- $03-2254$
}

Approved for public release; distribution is unlimited.
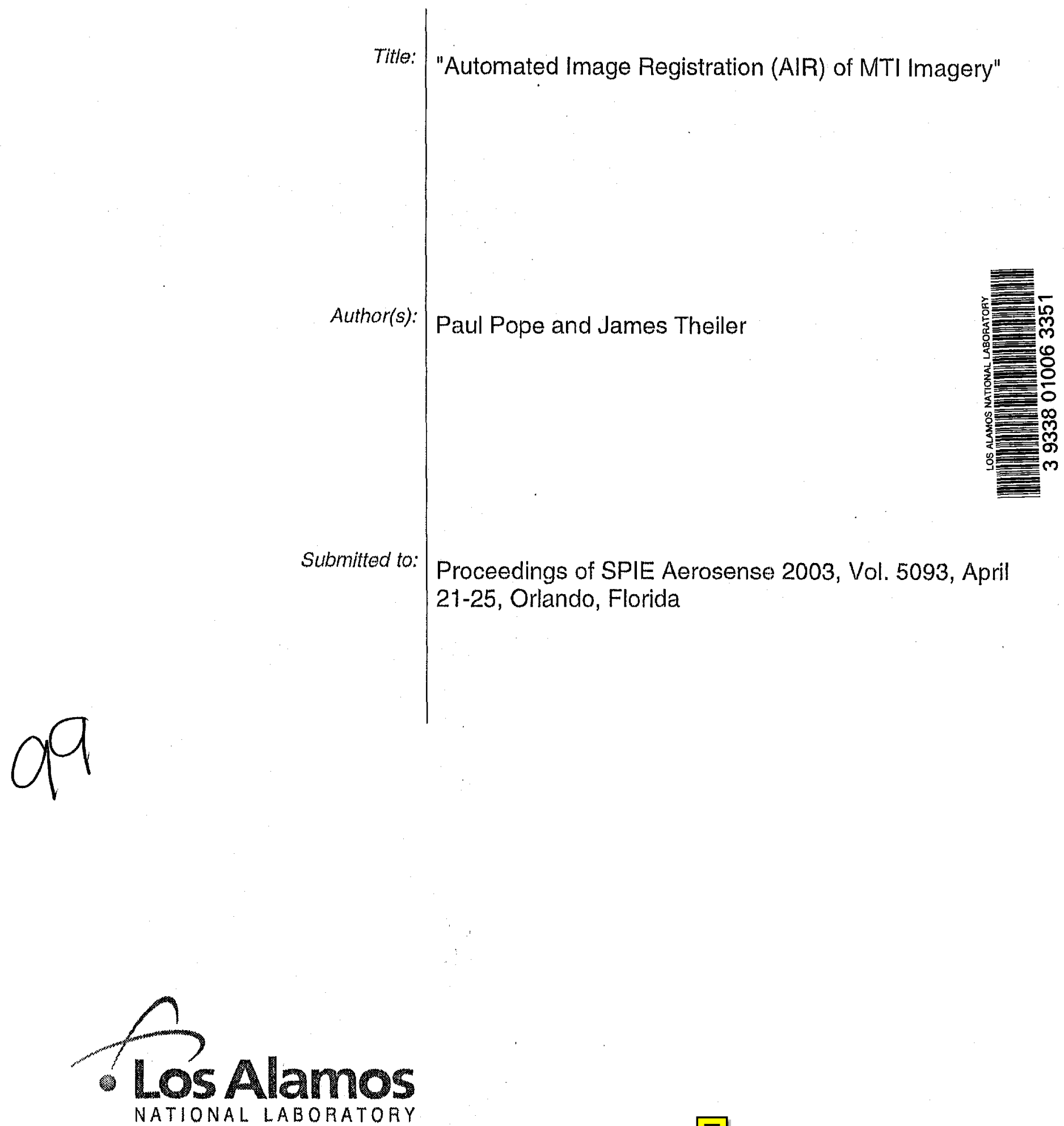

Los Alamos National Laboratory, an affirmative action/equal opportunity employer, is opera $\equiv$, the University of California for the U.S. Department of Energy under contract W-7405-ENG-36. By acceptance of this article, the pumoner recognizes that the U.S. Government retains a nonexclusive, royalty-free license to publish or reproduce the published form of this contribution, or to allow others to do so, for U.S. Government purposes. Los Alamos National Laboratory requests that the publisher identify this article as work performed under the auspices of the U.S. Department of Energy. Los Alamos National Laboratory strongly supports academic freedom and a researcher's right to publish; as an institution, however, the Laboratory does not endorse the vlewpoint of a publication or guarantee its technical correctness. 


\title{
Automated image registration (AIR) of MTI imagery
}

\author{
P. Pope and J. Theiler* \\ Space and Remote Sensing Sciences, Los Alamos National Laboratory, Los Alamos, NM 87545
}

\begin{abstract}
This paper describes an algorithm for the registration of imagery collected by the Multispectral Thermal Imager (MTI). The Automated Image Registration (AIR) algorithm is entirely image-based and is implemented in an automated fashion, which avoids any requirement for human interaction. The AIR method differs from the "direct georeferencing" method used to create our standard coregistered product since explicit information about the satellite's trajectory and the sensor geometry are not required. The AIR method makes use of a maximum cross-correlation (MCC) algorithm, which is applied locally about numerous points within any two images being compared. The MCC method is used to determine the row and column translations required to register the bands of imagery collected by a single SCA (band-to-band registration), and the row and column translations required to register the imagery collected by the three SCAs for any individual band (SCA-to-SCA registration). Of particular note is the use of reciprocity and a weighted least squares approach to obtaining the band-to-band registration shifts. Reciprocity is enforced by using the MCC method to determine the row and column translations between all pair-wise combinations of bands. This information is then used in a weighted least squares approach to determine the optimum shift values between an arbitrarily selected reference band and the other 15 bands. The individual steps of the AIR methodology, and the results of registering MTI imagery through use of this algorithm, are described.

Keywords: MTI, image, multispectral, registration, correlation, reciprocity, weighted least squares
\end{abstract}

\section{INTRODUCTION}

The Multispectral Thermal Imager (MTI) is a multispectral, pushbroom remote sensing instrument which collects imagery of the Earth's surface in 16 different wavelength bands (one of which is redundant). Imagery is acquired through the use of three Sensor Chip Assemblies (SCAs), each of which contains 16 linear CCD arrays (Fig. 1). The first SCA (SCA 1, also called the "singlet") provides "forward" viewing, while the other two SCAs (SCAs 2 and 3, also called the "doublet") provide "aft" viewing. The doublet images bracket the singlet image on the left and the right sides. There is a small amount of overlap, in the cross-track direction, between the area imaged by the singlet and those imaged by the doublet. The start and stop times for each band on each SCA are staggered such that the first scan line acquired by each band are of approximately the same line on the ground. The MTI satellite has cross-track and along-track pointing capability. Further information about the MTI satellite has been described elsewhere. 1,2

\subsection{Problem statement}

Raw MTI images cannot be used effectively because they are not coregistered. Coregistration means that a feature imaged in one band is located at the same row and column position in another band. This facilitates the use of these multispectral data to derive physical measurements which are useful in characterizing features within the imagery. Most of the useful applications of MTI imagery (e.g. estimation of ground temperature, extraction of spectral reflectance, and cartography) ${ }^{3}$ require that the bands of imagery output from each SCA be coregistered, and that these coregistered bands are then mosaicked into a single image stack. However, differences in viewing geometry between the three SCAs, as well as inconsistencies between the staggered timing and the viewing geometry at the time of image acquisition, present an interesting challenge to this requirement. 


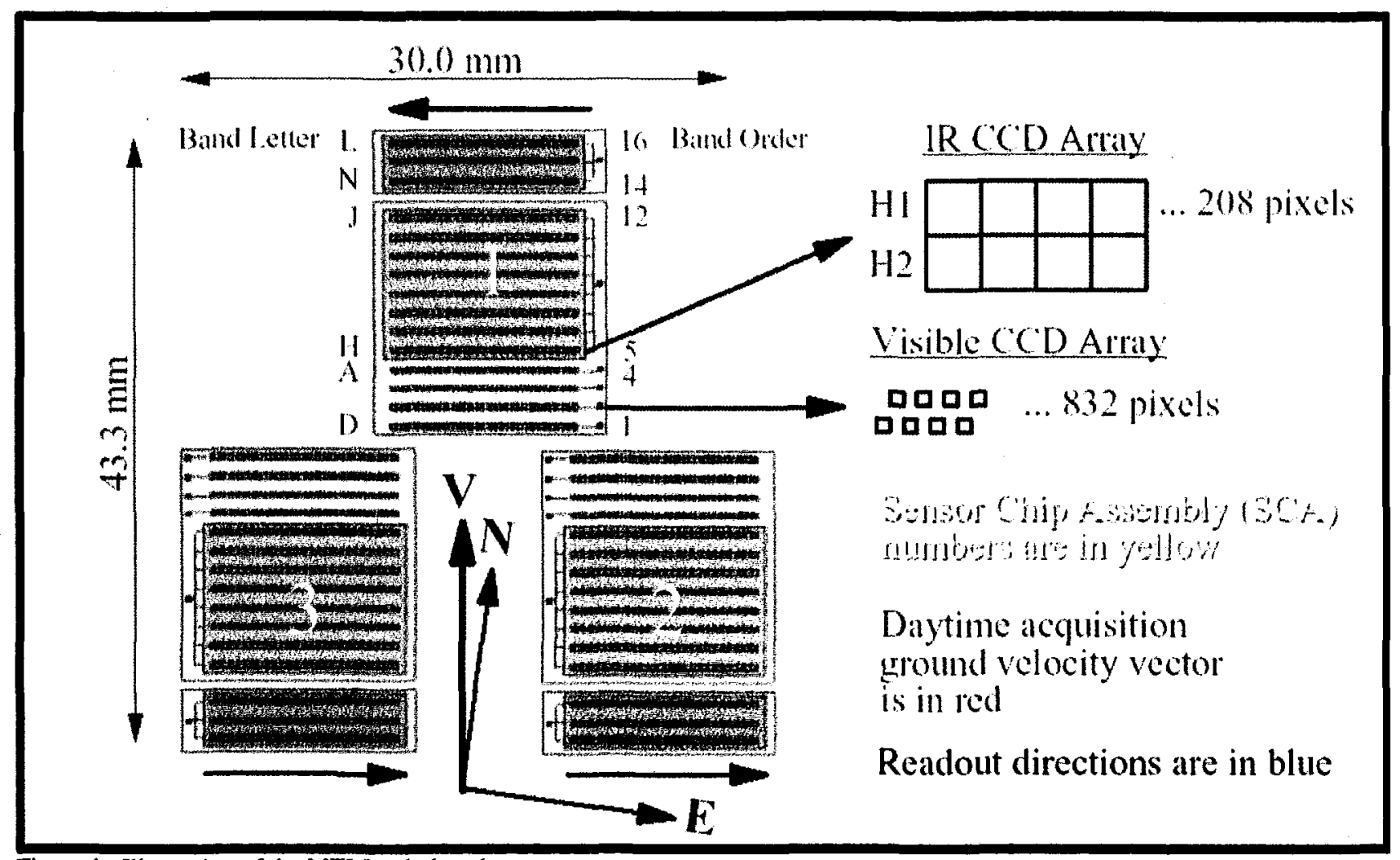

Figure 1. Illustration of the MTI focal plane layout.

We distinguish two types of misregistration. The misregistraton between imagery acquired by a particular SCA is called band-to-band error, while misregistration between a particular band of imagery from all three SCAs is called SCA-to-SCA error. The main source of both of these misregistration errors is the staggered timing used to acquire the first scan line from each band on each SCA. During a daytime overpass, imagery is acquired by starting band L on SCA 1 first. Other bands are then turned on sequentially according to their order on the focal plane array ("band order") . Inconsistencies between this staggered timing with the viewing geometry cause the first scan line acquired by different bands to lie along different lines on the ground.

\subsection{Proposed solution}

When nadir images are inspected through on-screen display, the bulk of the band-to-band and SCA-to-SCA misregistration errors appears as simple translations of the imagery. More complex effects do exist, due to differences in viewing geometry of the SCAs, parallax due to terrain relief, motion of the platform, etc. However, useful imagery is still obtained by correcting the bulk of the misregistration errors through application of simple translations. ${ }^{4}$ Also, it is desirable to perform this correction in an automated fashion such that there is no need for human intervention. This supports rapid image processing in a production environment, as well as ensuring repeatable and objective results. Finally, an entirely image-based technique is useful because it does not rely on ancillary data (e.g. spacecraft attitude and position), which may be unavailable due to equipment failure.

The proposed solution, called Automated Image Registration (AIR), is entirely image-based and is implemented in an automated fashion, which avoids any requirement for human interaction. Image-to-image matching is used to gather information about the band-to-band and SCA-to-SCA shifts. Since the images are multispectral, there are varying levels of correlation between different bands. This problem is addressed via optimization of the shift information through a weighted least squares technique. The final output is a single stack of 16 bands of imagery, in which the bands are coregistered, and the imagery from each SCA has been mosaicked together. The overall process is analogous to arranging sheets of paper into neat stacks. For each SCA, the bands of imagery are translated up and down, left and right, to create a single image stack composed of 16 coregistered bands. The SCA 2 and 3 image stacks are then placed 
such that they straddle and slightly overlap the SCA 1 image stack. This final arrangement yields the image stack output by the AIR method.

\section{METHODOLOGY}

The methodology used to create an AIR product is described next. First an overview of the process is provided. Next, the primary methods used in the AIR process are described. Finally, the use of these primary methods to perform band-to-band and SCA-to-SCA registration are described.

An image-based coordinate system is used in this research. The origin $(0,0)$ of this coordinate system is defined as the upper left corner of the upper left pixel. The i axis corresponds to rows in the image, and is oriented from the upper left corner to the lower left corner. The $j$ axis corresponds to columns in the image, and is oriented from the upper left corner to the upper right corner.

\subsection{Pre-processing}

There are several pre-processing steps which take the imagery from our standard LEVEL_1B_R product (i.e. spectrally calibrated, top-of-atmosphere radiances) and modify it into a form which can be used as input to the AIR process. The visible band images (bands A, B, C, and D) are deinterlaced by shifting alternate columns by 2 pixels. The visible bands are then resampled to equivalent IR-sized imagery (i.e. from $\approx 5 \mathrm{~m}$ GSD to $\approx 20 \mathrm{~m}$ GSD). Both the visible and IR images from SCA 1 are flipped and flopped, while those from SCA 2 and 3 are only flipped. Flipping refers to an interchange of rows about the middle row, while flopping refers to an interchange of columns about the middle column. Flipping and flopping are performed such that the images are approximately oriented in the cardinal directions (i.e. the top of the imagery is northward, the right side of the imagery is eastward, etc.) Since the LEVEL_1B_U product contains 48 images ( 3 SCAs times 16 bands of imagery per SCA), the final output is 48 IR-sized image stored within a working directory. The AIR method uses these 48 images as input to create the final image stack. The AIR method is currently restricted to processing imagery acquired during a daytime, nadir viewing overpass.

\subsection{Overview of the AIR method}

The overall process to create an AIR product proceeds as follows (Fig. 2). A loop over the three SCAs is performed. For each SCA, the following processes are performed. The band-to-band shifts in the $i$ and $j$ directions are calculated. These band-to-band shifts are optimized (by using a weighted least squares method described below). The optimized shift information is used to create an image stack. At the completion of the loop over the three SCAs, there are three image stacks, each of which consists of 16 coregistered bands. The SCA-to-SCA shifts in the $i$ and $j$ directions are determined between the SCA 1 and SCA 2 image stacks, as well as the SCA 3 and SCA 1 image stacks. These shifts are used to mosaic the three image stacks together to create the final output image stack.

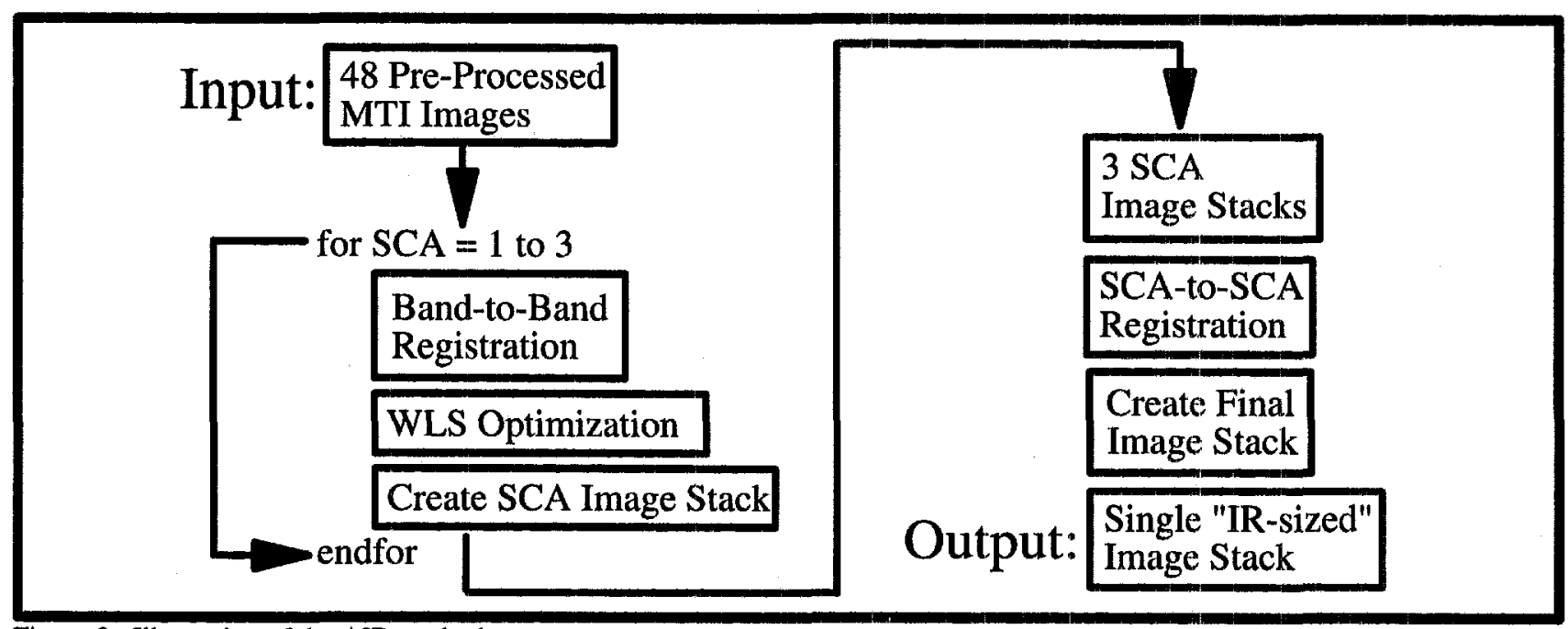

Figure 2. Illustration of the AIR method. 
Since the imagery is processed in a serial manner, a choice had to be made concerning the order of the shift corrections; perform band-to-band registration for each SCA to produce 3 image stacks containing 16 bands each, and then mosaic the 3 SCA image stacks, or mosaic the 3 SCA images for each band, and then perform band-to-band registration using the 16 SCA mosaics for each band. The former processing order was chosen based on the assumption that the SCA-to-SCA overlap is so small (approximately 40 pixels for nadir imagery) that a weak solution to the SCA-to-SCA mosaic for individual bands would compromise the band-to-band registration.

The processing currently proceeds in a modular fashion. Intermediate results are saved to harddisk, and then read in and processed by the next step in the processing chain. This facilitates error checking and experimentation with the method.

\subsection{Primary methods}

The primary information required to perform band-to-band and SCA-to-SCA registration are the shift amounts in both the $i$ and $j$ directions. The primary methods used by the AIR process to obtain this information are image-to-image matching and shift prediction. These primary methods are explained in sections 2.3.1 and 2.3.2.

\subsubsection{Image-to-image matching}

The image-to-image matching method used by the AIR process involves calculating the correlation between image subsections extracted from the two images being compared. This commonly used image-to-image matching technique is described in detail elsewhere 5,6 , so only a brief overview is provided here.

Consider two images for which the $\mathrm{i}$ and $\mathrm{j}$ misregistration shifts between them are to be determined. Image-to-image matching is used to determine the shifts in the $i$ and $j$ directions by comparing subsections from each of these images as follows (Fig. 3).

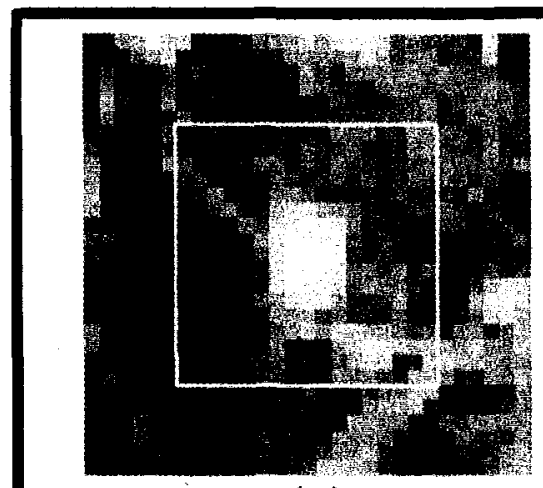

(a)

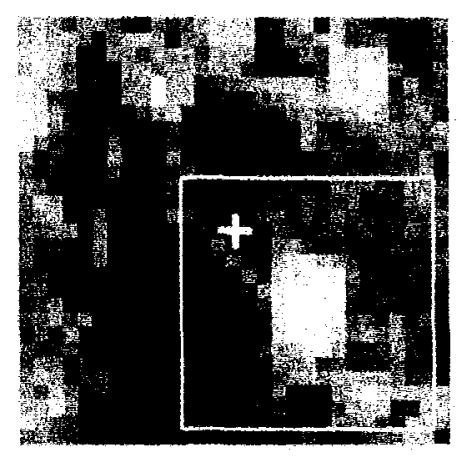

(b)

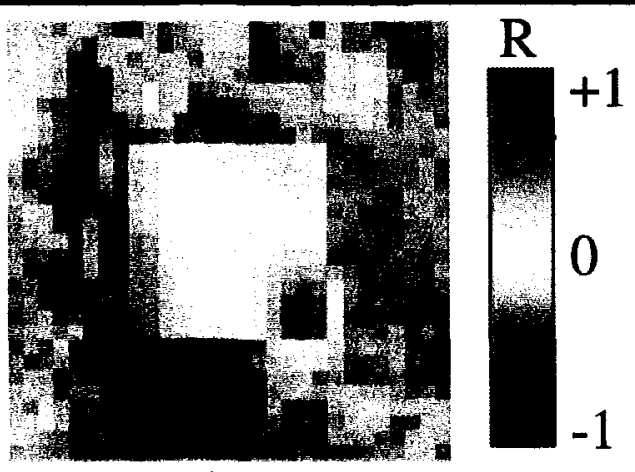

(c)

Figure 3. Illustration of image-to-image matching, showing the template image (a), the search window and a single search image (b), and the correlation image (overlaid on the search window) (c). The template and search images are 17 by 17 pixels, and the search window is 29 by 29 pixels (yellow squares). Position $\left(i_{1}, j_{1}\right)$ is shown by a " + " in (a), and position ( $\left.i_{2}, j_{2}\right)$ is shown by a " + " in (b). The true shifted location of the bright feature in (a) is 5 pixels in the $i$ direction and 5 pixels in the $j$ direction, and is shown by an " $x$ " in (b).

A subsection of size $t_{m}$ rows by $t_{n}$ columns (where $m$ and $n$ are odd integers) is taken from a position centered at $\left(i_{1}, j_{1}\right)$ in the first image (Fig. 3(a)). This subsection is called the "template image". This raster of digital numbers (DNs) is represented by " $t$ ", and the digital number at position $(i, j)$ is represented by $t(k)$, where $k$ is the "pixel index", given by,

$$
k=t_{m} i+j
$$

A larger subsection, consisting of $S_{m}$ rows by $S_{n}$ columns (where $S_{m}$ and $S_{n}$ are odd integers, and $S_{m}>t_{m}$ and $S_{n}>t_{n}$ ) is taken from a position centered at $\left(\mathrm{i}_{2}, \mathrm{j}_{2}\right)$ in the second image (Fig $3(\mathrm{~b})$ ). This subsection is called the "search window". The main assumption is that the same (or a very similar) template image extracted from the first image lies somewhere within the search window. To find this position within the search window, subsections of size $t_{m}$ rows by $t_{n}$ columns are taken from sequential positions from within the search window. Each of these subsections is called a 
"search image". This raster of DNs is represented by "s", and the digital number at position $(i, j)$ is represented by $s(k)$. The metric used to determine the similarity between the template and search images is the normalized cross correlation coefficient, $R$. The normalized cross correlation coefficient is given by,

$$
R=\frac{\sum_{k=0}^{k}\left(t(k)-\mu_{t}\right)\left(s(k)-\mu_{s}\right)}{\left[\sum_{k=0}^{k}\left(t(k)-\mu_{t}\right)^{2} \sum_{k=0}^{k}\left(s(k)-\mu_{s}\right)^{2}\right]^{1 / 2}}
$$

where $\mu_{t}$ is the mean of the DNs in the template image, $\mu_{s}$ is the mean of the DNs in the search image, and $K$ is equal to the maximum pixel index value, given by,

$$
K=t_{n} t_{m}-1
$$

where $t_{n}$ times $t_{m}$ is equal to the total number of pixels in the template image (as well as the search image). The normalized cross correlation coefficient is calculated between the template image and each search image. This comparison creates a "correlation image", or "correlation surface" (Fig. 3(c)).

Since the two images being compared are not (in general) perfectly correlated, "high" (or "strong") correlation values must be identified. This is achieved by applying a threshold to the correlation image (Fig. 4). Regions of strong positive correlation can be identified by applying a threshold which is greater than zero, but less than or equal to 1 . Regions of strong negative correlation can be identified by applying a threshold which is less than zero, but greater than -1 . Locations within the correlation image which meet the threshold criterion are used to calculate summary statistics (i.e. mean, median, and standard deviation) in both the $i$ and $j$ directions. Finally, the location of the template image within the search window can be inferred from these summary statistics. For example, the mean value in the $i$ and $j$ directions could be used as to be the best estimate of this location, and the standard deviation of the mean in the $i$ and $j$ directions could be used as a measure of the accuracy of this location.

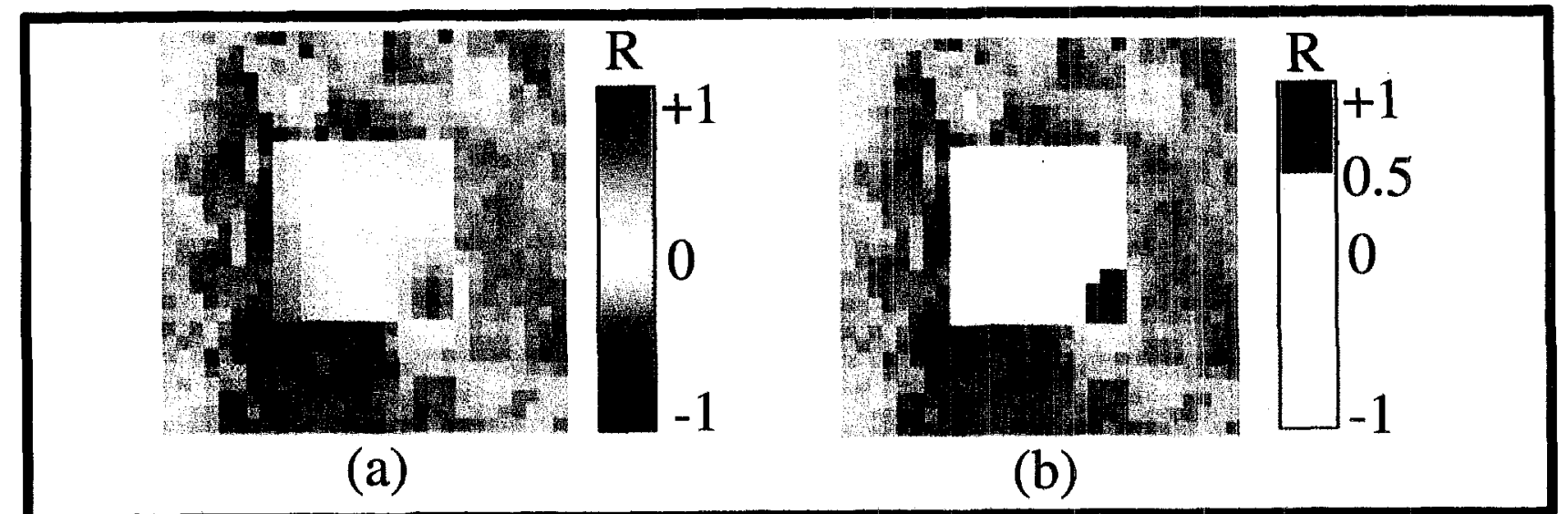

Figure 4. Illustration of applying a correlation threshold. A threshold is applied to the correlation image (a) such that a region of strong correlation (i.e. $R \geq 0.5$ ) has been identified (b). The estimated shifts in the $i$ and $j$ directions are $5.6 \pm 1.1$ pixels and $5.1 \pm 0.8$ pixels, respectively. 
The correlation image formed between different bands of imagery depends on the positions from which the template image and search window are extracted. Therefore, the correlation threshold criterion may fail for areas where there is no strong correlation between the images. To avoid this situation as much as possible, the image-to-image matching process described above is conducted at numerous places within each image. This allows the shift between the images to be determined at several places. Summary statistics are calculated from the shift information gathered from places where the correlation threshold is successful. These summary statistics are used to determine an overall shift between the two images, as well as the quality (accuracy) of this information. If the correlation threshold is not met at any position, the overall shift value is set to zero and the error is set to a relatively high value (e.g. 100 pixels). Applying image-to-image matching at numerous places in the imagery is referred to in this paper as the "maximum cross correlation" (MCC) method. 5

\subsubsection{Reciprocity}

The previous section has described how image-to-image matching is performed between two images by comparing the similarity between template and search images through creation of a correlation image. Consider the correlation image which would be formed if the image from which the template was extracted, was exchanged with the image from which the search window was extracted. This interchanging (or swapping) of images is called reciprocity (Fig. 5). The correlation image created under this reciprocity scenario will not be exactly the same as the first correlation image. The correlation images will only have the same value at their centers. This is due to the fact that the imagery contained within the search windows extracted from the two images are not the same. However, it is important to note that no matter which order is used to compare the two images, the shift between them is the same in magnitude. Therefore, it is useful to calculate both correlation images since each of them provide separate estimates of the same quantity of interest.

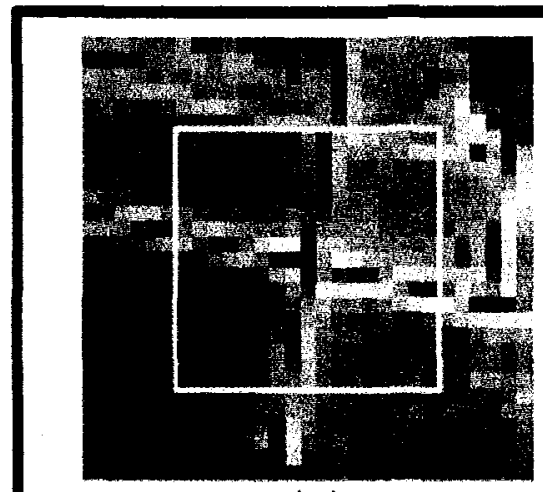

(a)

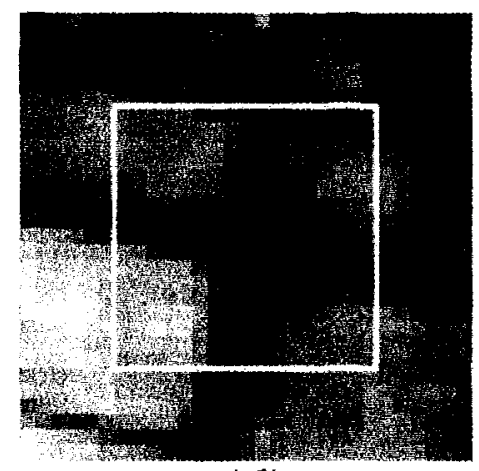

(d)

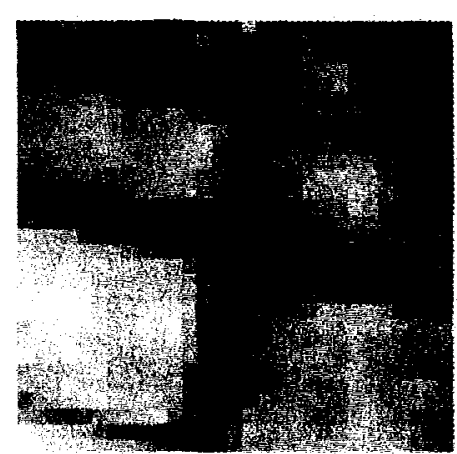

(b)

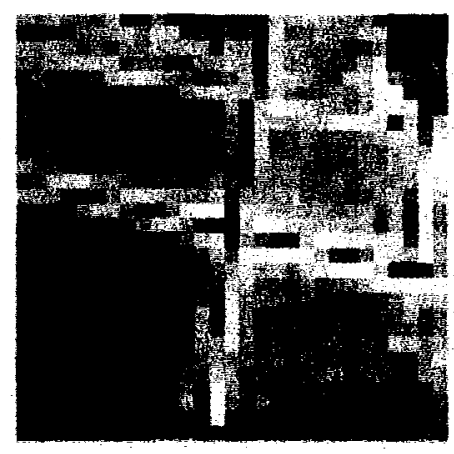

(e)

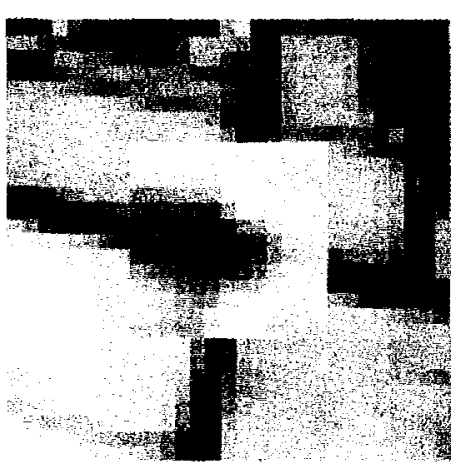

(c)
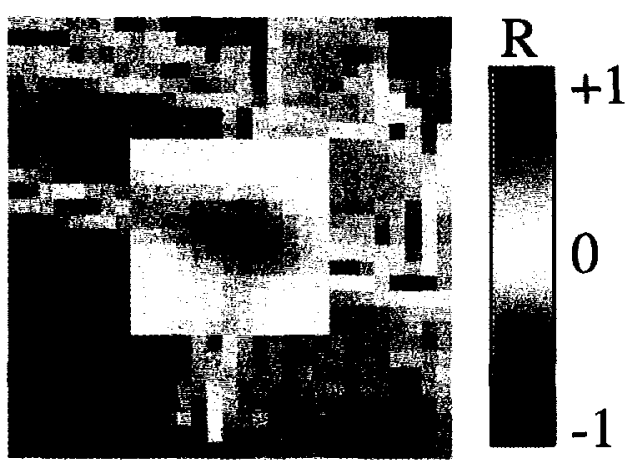

(f)

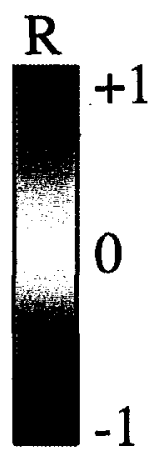

\section{1}

Figure 5. Illustration of reciprocity. In the first scenario, a template image is extracted from band D (a) and cross correlated with a search window from band $L$ (b) to create a correlation image (c). The bands are then interchanged, such that a template image is extracted from band $L(d)$ and cross correlated with a search window from band $D(e)$ to create a correlation image (f). Note that the correlation images are not exactly the same. Only the center values are equal. 


\subsubsection{Weighted Least Squares}

In the process of designing the AIR method, a means to optimize the shift information gathered by the MCC method was sought. It seemed likely that Weighted Least Squares (WLS) could be used to leverage the redundant shift information provided by reciprocity, as well as the error measures returned by the MCC method. The WLS technique involves the simultaneous fitting of "observations" to "observation equations." Therefore, a definition of an individual shift observation, and the general observation equation which connects all the shift observations together, must be described. The following explanation formulates the necessary equations in terms of i shift information gathered by the MCC technique. Analogous equations can also be written in terms of $\mathbf{j}$ shift information.

Consider two bands, $\mathrm{p}$ and $\mathrm{q}$, for which i shift information has been gathered by the MCC technique. The i shift observation, $\Delta \mathrm{i}_{\mathrm{pq}}$, can be expressed as,

$$
\Delta \mathrm{i}_{\mathrm{pq}}=\mu \mathrm{i}_{\mathrm{pq}} \pm \sigma \mathrm{i}_{\mathrm{pq}}
$$

where $\mu \mathrm{i}_{\mathrm{pq}}$ is a measure of central tendency (e.g. the mean or median), $\sigma \mathrm{i}_{\mathrm{pq}}$ is a measure of error (e.g. standard deviation or standard deviation of the mean), $1 \leq \mathrm{p} \leq \mathrm{B}, 1 \leq \mathrm{q} \leq \mathrm{B}$, and $\mathrm{B}$ is the total number of bands. An observation equation which utilizes this shift information may be written as,

$$
\Delta \mathrm{i}_{\mathrm{pq}}=\Delta \mathrm{i}_{\mathrm{q} 0}-\Delta \mathrm{i}_{\mathrm{p} 0}
$$

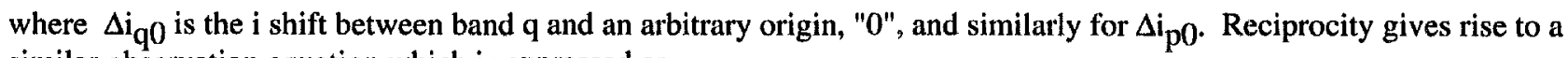
similar observation equation which is expressed as,

$$
\Delta \mathrm{i}_{\mathrm{qp}}=\Delta \mathrm{i}_{\mathrm{p} 0}-\Delta \mathrm{i}_{\mathrm{q} 0}
$$

Note that in theory $\Delta \mathrm{i}_{\text {gp }}$ should be equal to $-\Delta \mathrm{i}_{\mathrm{pq}}$. However, as explained previously, the numerical values gathered by the MCC technique will not yield the reciprocity condition perfectly. The total number of observation equations which can be formed using Eq. (5) is equal to $B^{2}$. However, if $q=p$, then the observation equation becomes,

$$
\Delta \mathrm{i}_{\mathrm{pp}}=\Delta \mathrm{i}_{\mathrm{p} 0}-\Delta \mathrm{i}_{\mathrm{p} 0}
$$

which does not yield useful information about the relative shifts between different bands. The number of equations which can be formed using Eq. (7) is equal to the number of bands, B. Therefore, the total number of useful observation equations, $\mathbf{B}^{\prime}$, is equal to,

$$
\mathrm{B}^{\prime}=\mathrm{B}^{2}-\mathrm{B}
$$

Since $B=16$ for MTI, then $B^{\prime}=240$. Therefore, 240 observations must be gathered by the MCC method and 240 observation equations must be formed for each SCA.

When solving numerically for the optimized shift parameters, the measure of central tendency, $\mu \mathrm{i}_{\mathrm{pq}}$, is used in place of the $\Delta \mathrm{i}_{\mathrm{pq}}$ value in Eq. (5). The error in this estimate of the $\Delta \mathrm{i}_{\mathrm{pq}}$ value can be used to weight the observation equations. The weight for each observation equation is given by,

$$
w_{p q}=1 /\left(\sigma i_{p q}\right)^{2}
$$

The system of equations, in matrix notation, relating the observations, observation equations, and weights is given by,

$$
W A x=W d
$$


where $\mathrm{X}$ is a $(\mathrm{B} \times 1)$ vector equal to,

$$
x=\left[\Delta i_{10}, \Delta i_{20}, \ldots, \Delta i_{B 0}\right]^{T}
$$

$d$ is a $\left(B^{\prime} \times 1\right)$ vector (which contains the shift measurements) equal to,

$$
\mathrm{d}=\left[\Delta \mathrm{i}_{12}, \Delta \mathrm{i}_{21}, \ldots, \Delta \mathrm{i}_{\mathrm{pq}}, \Delta \mathrm{i}_{\mathrm{qp}}, \ldots\right]^{\mathrm{T}}
$$

$A$ is a $\left(B^{\prime} \times B\right)$ matrix of coefficients from the observation equation (i.e. $1,-1$, or 0$)$, and $W$ is a $\left(B^{\prime} \times B^{\prime}\right)$ diagonal matrix, where the diagonal values are equal to the weights (Eq. (9)) for each observation equation.

The final WLS solution for the optimized shifts, in matrix notation, is given by,

$$
x=\left[A^{T} W A\right]^{-1}\left[A^{T} W d\right]
$$

The actual numerical solution is obtained through singular value decomposition. Once the $\Delta \mathrm{i}_{\mathrm{p} 0}$ values have been solved for, then Eq. (5) can be used to determine the optimum shift between any two bands, $p$ and $q$.

\subsubsection{Shift prediction}

The image-to-image matching explained previously works best (in terms of accuracy and computational efficiency) if the search window is kept relatively small. This can be accomplished if the center of the search window center, $\left(i_{2}, j_{2}\right)$, is placed close to the location where strong correlations are expected. This means that the major portion of the shift between two images must be predictable. It was observed that the bulk of the shift magnitudes in the $i$ and $j$ directions can be estimated via linear regression (Fig. 6). This is possible since the band-to-band and SCA-to-SCA shifts are caused by the staggered start times. Since the start times are a function of band order, then the shift amounts are also a function of band order. Therefore, linear regression is used to fit a separate line through the $i$ and $j$ shifts as a function of band order. The location of the center of the search window can then be calculated via,

$$
\mathrm{i}_{2}=\mathrm{i}_{1}+\mathrm{i}_{0}
$$

and

$$
\mathrm{j}_{2}=\mathrm{j}_{1}+\mathrm{j}_{0}
$$

where $\mathrm{i}_{0}$ and $\mathrm{j}_{0}$ are the shifts estimated by the linear shift prediction equations. The shift prediction equations are initialized by using image-to-image matching, with relatively large search windows, between visible bands A, B, and C. The search window size is based on the expected range of $i$ and $j$ shifts which will be encountered. Bands $A, B$, and $C$ are used since they can be expected to exhibit strong correlations over a wide range of land covers. The linear regression parameters (slope and intercept) are recalculated as more shift information is gathered. 


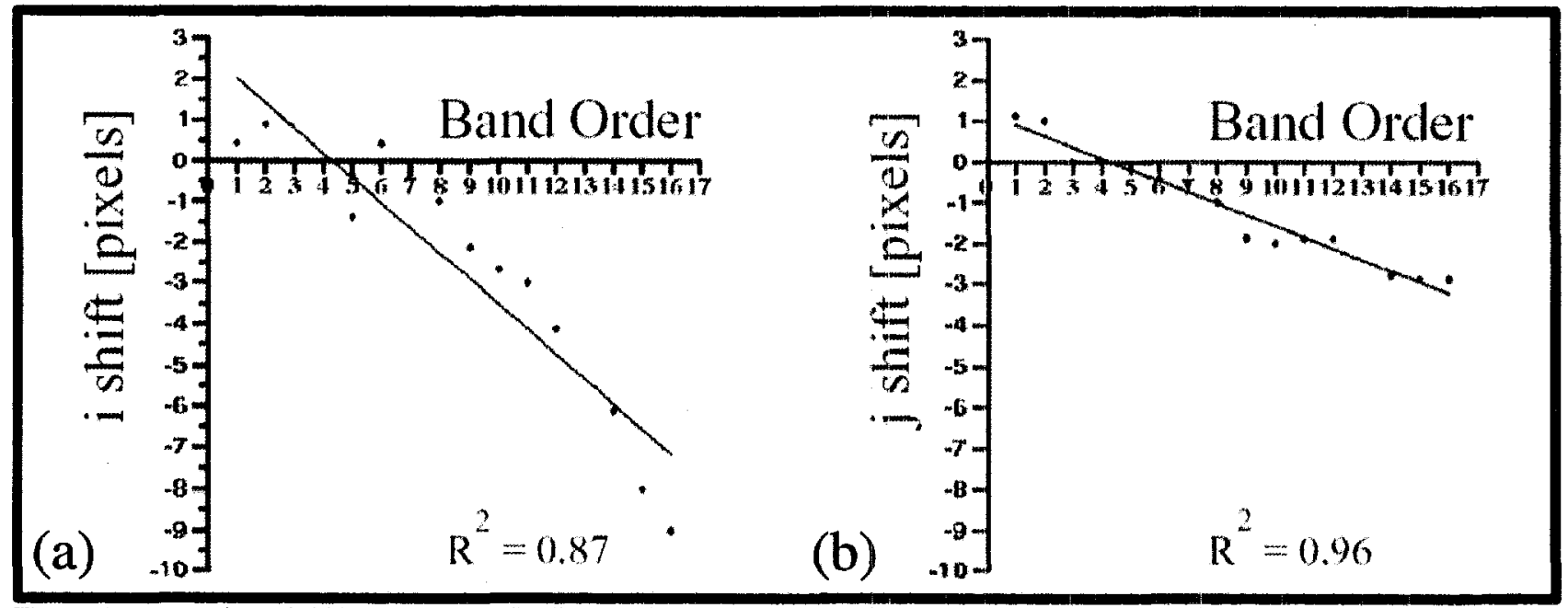

Figure 6. Illustration of shift prediction. The linear shift prediction equation for the band-to-band i shifts (a) and $j$ shifts (b) (for SCA 1 of a typical image) are shown. Note that the i shifts are much larger than the $j$ shifts.

\subsection{Band-to-band registration}

The band-to-band registration process takes the individual images for each band of a single SCA and creates an image stack of coregistered bands. Therefore, the outputs from this process are three image stacks, one for each SCA, each consisting of 16 coregistered bands.

The parameters used to guide the MCC method in determining the band-to-band shifts are currently set as follows: fifty samples distributed over the image via systematic random sampling 7 , a correlation threshold of less than -0.5 and greater than 0.5 (i.e. both strong positive and strong negative correlations are allowed to pass), a template size of 17 rows by 17 columns, a maximum i shift of 30 pixels, and a maximum j shift of 5 pixels. All bands are coregistered to the image-based coordinate system defined by band A. The MCC method is applied to all pair-wise combinations of bands (i.e. 240 combinations). The median value and standard deviation, in each direction, are used as the overall shift value and the measure of error, respectively.

\subsection{SCA-to-SCA registration}

Even though the bands within the SCA image stack have been coregistered by the previous process, residual band-to-band error is still present. This residual band-to-band error could adversely affect the determination of the SCA-to-SCA shifts. Therefore, the SCA-to-SCA shift is determined for each band. The median value, in each direction, is used as the final SCA-to-SCA shift value. The output of this process is a mosaic of the three SCA image stacks (i.e. an AIR product).

The parameters used to guide the MCC method in determining the SCA-to-SCA shifts are currently set as follows: fifty samples distributed within the overlap area of each SCA via systematic sampling 7 , a correlation threshold of greater than 0.9 (i.e. only strong positive correlations are allowed to pass), a template size of 21 rows by 7 columns, a maximum i shift of 30 pixels, and a maximum $j$ shift of 5 pixels. All SCA image stacks are coregistered to the image-based coordinate system defined by the SCA 3 image stack.

\section{ACCURACY ASSESSMENT}

An accuracy assessment was performed to investigate the capability of the AIR method to determine the band-to-band and SCA-to-SCA shifts in both the $i$ and $j$ directions as compared to manual determination of these values. The test imagery used, gathering of reference data, testing methodology, and results of the accuracy assessment are discussed next. 


\subsection{Test imagery}

Twenty MTI images were randomly selected from a subset of the entire MTI image database. The following criteria

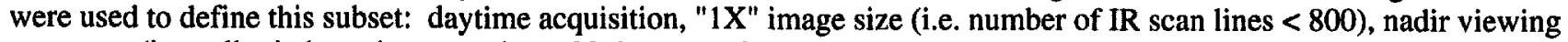
geometry (i.e. roll, pitch, and yaw angles $<20$ degrees), date of acquisition after "TCAL failure" (11/01/2000) and before "sun exposure" (11/12/2001), minimal cloud cover (< 10 percent), land only (no coastline nor open ocean imagery), and an equal number of "humid" and "arid" sites. An AIR product was created for each of these test images. Each image took approximately 23 minutes to process.

\subsection{Reference data}

Reference data about the band-to-band and SCA-to-SCA shifts in both the $i$ and $j$ directions for each of the test images was gathered by manually defining conjugate points. The band-to-band shift reference data were determined as follows. For each SCA, the 16 bands were displayed next to the band A image. A single, point-like feature was identified in both images. The location of this feature in both images was recorded. The difference between these locations, in both the $i$ and $\mathrm{j}$ directions, was used as the "true" (or "reference") shift. Therefore, each SCA required that 16 conjugate points be defined. The SCA-to-SCA shift reference data was determined as follows. The manually determined band-to-band shifts were used to create an image stack for each SCA. For each SCA overlap pair (i.e. SCA 1 to SCA 2 and SCA 3 to SCA 1), the band D images from each SCA stack were displayed next to each other. A single, point-like feature was identified in both images. The location of this feature in both images was recorded. The difference between these locations, in both the $i$ and $j$ directions, was used as the reference shift. Therefore, each SCA overlap pair required that one conjugate point be defined. Note that a total of 50 conjugate points were required for each image. Since 20 test images were used, the creation of the reference shift data set required the manual definition of 1000 conjugate points.

A brief discussion of the manually determined band-to-band and SCA-to-SCA shifts gathered from the test images provides insight into how the MCC parameter values were chosen. The range of band-to-band shifts was less than 30 pixels in the $i$ direction and less than 5 pixels in the $j$ direction. The range of SCA-to-SCA shifts was of the same magnitude as the band-to-band shifts.

\subsection{Pre-WLS vs. post-WLS band-to-band shifts}

The usefulness of the WLS technique described previously was tested. The RMSE between the AIR and the manually determined band-to-band shifts was calculated before and after application of the WLS adjustment. The RMSE was calculated separately for each SCA and for each direction. The RMSE was used as the error metric since it provides a single measure of accuracy by combining the residual errors for all 16 bands.

Statistical tests were conducted to make objective inferences about the utility of the WLS technique. The post-WLS RMSE in the i direction was significantly less than the non-WLS RMSE for all three SCAs (p-value $<0.01$ ). The post-WLS RMSE in the j direction was significantly less than the non-WLS RMSE for SCAs 2 and 3 (p-value $<0.01$ ). The post-WLS RMSE in the $j$ direction was not significantly less than the non-WLS RMSE for SCA 1 (p-value $>0.01$ ). The summary statistics (mean and standard deviation of the mean) are shown in Table 1.

\begin{tabular}{|l|c|c|c|}
\hline & SCA 1 & SCA 2 & SCA 3 \\
\hline RMSE difference (i shifts) & $-0.3 \pm 0.06$ pixels & $-0.2 \pm 0.06$ pixels & $-0.3 \pm 0.05$ pixels \\
\hline RMSE difference (j shifts) & $-0.04 \pm 0.02$ pixels & $-0.2 \pm 0.05$ pixels & $-0.1 \pm 0.03$ pixels \\
\hline
\end{tabular}

Table 1. Summary statistics for the pre-WLS vs. post-WLS band-to-band shifts accuracy assessment.

Even though the effect of using the WLS method to "optimize" the shift values was statistically significant, its functional (practical) significance needs to be addressed. The values in Table 1 show that the WLS method was able to consistently reduce the RMSE by a subpixel amount. Since subpixel band-to-band registration is desirable, this technique should prove useful in other studies involving the registration of remotely sensed multispectral imagery. However, the computational load required to gather the necessary input information for the WLS method must be taken into account.

\subsection{Reference vs. AIR band-to-band shifts}

The accuracy of the AIR band-to-band shifts was determined relative to the manually derived reference data. Statistical tests were conducted to make objective inferences about the utility of the AIR method to determine band-to-band shifts. 
The RMSE, in both the $i$ and $j$ directions, was found to be significantly different from zero for all SCAs (p-value $<0.01$ ). The summary statistics (mean and standard deviation from the mean) are shown in Table 2.

\begin{tabular}{|l|c|c|c|}
\hline & SCA 1 & SCA 2 & SCA 3 \\
\hline RMSE difference (i shifts) & $0.5 \pm 0.08$ pixels & $0.7 \pm 0.03$ pixels & $0.7 \pm 0.05$ pixels \\
\hline RMSE difference (j shifts) & $0.7 \pm 0.1$ pixels & $0.5 \pm 0.07$ pixels & $0.5 \pm 0.05$ pixels \\
\hline
\end{tabular}

Table 2. Summary statistics for the reference vs. AIR band-to-band shifts accuracy assessment.

Even though the $i$ and $j$ shifts as determined by the AIR method were found to be significantly different from those determined manually, the practical significance of this result must be addressed. The mean RMSE values in Table 2 range from 0.5 to 0.7 pixels. Therefore, the AIR method is able to automatically determine, to within 1 pixel, the same band-to-band shift values as can be determined manually.

\subsection{Reference vs. AIR SCA-to-SCA shifts}

The accuracy of the SCA-to-SCA shifts derived by the AIR method was determined relative to the manually derived reference data. Statistical tests were conducted to make objective inferences about the utility of the AIR method to determine SCA-to-SCA shifts. The difference in shifts, in both the $i$ and $j$ directions, was not significantly different from zero for both pairs of SCAs (p-value $<0.01$ ). The summary statistics (mean and standard deviation from the mean) are shown in Table 3 .

\begin{tabular}{|c|c|c|}
\hline & SCA 1 to SCA 2 & SCA 3 to SCA 1 \\
\hline Difference in i shifts & $0.4 \pm 0.2$ pixels & $0.5 \pm 0.2$ pixels \\
\hline Difference in $j$ shifts & $0.3 \pm 0.2$ pixels & $-0.5 \pm 0.2$ pixels \\
\hline
\end{tabular}

Table 3. Summary statistics for the reference vs. AIR SCA-to-SCA shifts accuracy assessment.

Once again, the practical significance of these results needs to be addressed. The fact that the difference in shifts was not significantly different from zero indicates that the AIR method was able to determine the SCA-to-SCA shift values to the same degree of accuracy as could be achieved manually. The summary statistics in Table 3 indicate that the error range is -0.5 pixels to 0.5 pixels. Therefore, the AIR method is able to determine, in an automated fashion, the SCA-to-SCA shifts to sub-pixel accuracy. It should be noted here that the summary statistics in Table 3 are inflated slightly, since these SCA-to-SCA shift errors include the band-to-band errors from the previous step (Table 2).

On-screen animation was also used as a qualitative assessment of the AIR method's ability to perform band-to-band and SCA-to-SCA registration as compared to manual registration. The bands of imagery contained in the final image stacks produced by using (1) AIR and (2) the manually derived shifts were displayed side-by-side and animated ("flickered"). It was observed that the AIR method was able to register the imagery to approximately the same degree of accuracy as manual registration, and for some imagery the AIR method appeared to be more accurate than the manually determined registration. Therefore, the numerical accuracy assessment results reported above are limited to expressing a relative accuracy rather than an absolute accuracy.

\section{CONCLUSIONS AND FUTURE RESEARCH}

An automated method to perform band-to-band and SCA-to-SCA registration of imagery acquired by the MTI sensor has been successfully developed and tested. The AIR method can create an image stack whose band-to-band and SCA-to-SCA registration accuracy is comparable to what can be accomplished through manual registration. Of particular note is the development of a weighted least squares technique which can optimize the shift information gathered by image-to-image matching.

Future research should address some remaining issues as well as test potential improvements to the method described here. A discussion of these, in order of increasing level-of-effort, follows. Of immediate use would be to modify the pre-processing step such that the IR bands are resampled to equivalent visible-sized bands. ${ }^{8}$ Although this would increase the storage requirement for each IR band by a factor of sixteen, the advantage would be to retain the spatial 
resolution of the visible bands. Only minor modifications to the AIR method would be required to enable the processing of visible-sized imagery (e.g. a larger search window size). Also, the AIR method should be modified to enable the processing of imagery acquired during a nighttime overpass. Another means of weighting the observation equations of the WLS technique would be to use the correlation information gathered by the image-to-image matching. Cloudy images might be handled by using a cloud mask to modify the distribution of the image-to-image sampling pattern. The AIR method currently produces three SCA stacks of 16 coregistered bands, and then mosaics these SCA stacks to create the final image stack. This approach was taken since the calibration information is different for each band of each SCA, so it was anticipated that the individual SCA image stacks might be useful for certain applications (e.g. small area of interest imaged by a single SCA). However, it might also be useful to sum the band-to-band and SCA-to-SCA shifts to derive a single shift which could be used to create the final image stack directly from the pre-processed imagery. This would skip the intermediate SCA image stack output entirely. Finally, the MCC technique might also be useful to "tweak" the band-to-band and SCA-to-SCA registration of the standard coreg product. 9

\section{ACKNOWLEDGEMENTS}

We have benefitted from the support and advice of the entire MTI team, but we particularly wish to acknowledge our colleagues Chris Borel (for suggesting the 2 pixel shift to de-interlace the visible bands), Keri Ramsey (for help with understanding the format of the IEVEL_1B_U product), Kim Pollock (for help in accessing the MTI database), Amy Galbraith (for useful discussions of spectral resampling issues), Santiago Ortega (for various insights into gathering conjugate point information), and Emily Riddle (for useful discussions of registration issues). This work was supported by the U.S. Department of Energy.

\section{REFERENCES}

1. P. G. Weber, B. C. Brock, A. J. Garrett, B. W. Smith, C. C. Borel, W. B. Clodius, S. C. Bender, R. R. Kay, and M. J. Decker, "Multispectral Thermal Imager mission overview," Proc. SPIE 3750, pp. 340-346, 1999.

2. J. J. Szymanski, W. Atkins, L. Balick, C. C. Borel, W. B. Clodius, W. Christensen, A. B. Davis, J. C. Echohawk, A. Galbraith, K. Hirsch, J. B. Krone, C. Little, P. Maclachlan, A. Morrison, K. Pollock, P. Pope, C. Novak, K. Ramsey, E. Riddle, C. Rohde, D. Roussel-Dupré, B. W. Smith, K. Smith, K. Starkovich, J. Theiler, and P. G. Weber, "MTI science, data products and ground data processing overview," Proc. SPIE 4381, pp. 195-203, 2001.

3. C. C. Borel, W. B. Clodius, A. B. Davis, B. W. Smith, J. J. Szymanski, J. Theiler, P. V. Villeneuve, and P. G. Weber, "MTI core science retrieval algorithms," Proc. SPIE 3753, pp. 403-415, 1999.

4. J. Smith, S. Motomatsu, J. G. Taylor, K. J. Jefferson, and B. R. Stallard, "MTI Transiation-only Registration," in Multispectral Thermal Imager (MTI) Symposium, U.S. Department of Energy, Office of Nonproliferation and National Security, Albuquerque, NM, March 12-13, 2001.

5. J. Gao and M. B. Lythe, "The maximum cross-correlation approach to detecting translational motions from sequential remote-sensing images," Computers and Geosciences 22, pp. 525-534, 1996.

6. J. A. Richards, Remote Sensing Digital Image Analysis, pg 67, Springer-Verlag, Berlin, Germany, 1993.

7. L. H. Keith, Environmental Sampling and Analysis: A Practical Guide, pp. 16-19, CRC Press, Boca Raton, FL, 1991.

8. J. S. Salazar, J. L. Smith, "Nonparametric image segmentation approach applied to MTI data," Proc. SPIE 5093, 2003. In this volume.

9. J. Theiler, A. Galbraith, P. Pope, K. Ramsey, and J. Szymanski, "Automated coregistration of MTI bands," Proc. SPIE 4725, pp. 314-327, 2002. 